\title{
Existence of solutions for fractional impulsive neutral functional infinite delay integrodifferential equations with nonlocal conditions
}

\author{
A. Anguraj ${ }^{a, *}$, M. Latha Maheswari ${ }^{b}$ \\ ${ }^{a}$ Department of Mathematics, P. S. G. College of Arts and Science, Coimbatore-641 014, Tamil Nadu, India \\ ${ }^{b}$ Department of Mathematics with CA, P. S. G. College of Arts and Science, Coimbatore-641 014, Tamil Nadu, India \\ This paper is dedicated to Professor Ljubomir Ćirić \\ Communicated by Professor V. Berinde
}

\begin{abstract}
This paper is mainly concerned with the existence of solutions for fractional impulsive neutral functional integrodifferential equations with nonlocal initial conditions and infinite delay. The results are obtained by the fixed point theorem. (C)2012. All rights reserved.

Keywords: Existence of solution, Fractional, Integrodifferential equations, Impulsive conditions, Nonlocal conditions, Fixed point theorem.

2010 MSC: primary 34A37, 34K37.
\end{abstract}

\section{Introduction}

Fractional Calculus deals with the generalization of integrals and derivatives of noninteger order. Fractional calculus involves a wide area of applications by bringing into a broader paradigm concepts of physics, mathematics and engineering [11, 13. Infact fractional differential equation is considered as an alternative model to nonlinear differential equations [8]. In [2, 12, the authors have proved the existence of solutions of abstract fractional differential equations by using fixed point tecniques. In consequence, the subject of fractional differential equations is gaining much importance and attention. For details, see [14, 15, 16] and the

\footnotetext{
${ }^{*}$ Corresponding author

Email addresses: angurajpsg@yahoo.com (A. Anguraj), lathamahespsg@gmail.com (M. Latha Maheswari)
} 
references therein. Subsequently several authors have discussed the problem for different types of nonlinear differential and integro differential equations including functional differential equations in Banach spaces.

The theory of impulsive differential equations has undergone rapid development over the years and played a very important role in modern applied mathematical models of real processes arising in phenomena studied in physics, population dynamics, chemical technology and economics. In [1, 7], Benchohra et al. established sufficient conditions for the existence of solutions for a class of initial value problems for impulsive fractional differential equations involving the Caputo fractional derivative of order $0<q \leq 1$ and $1<q \leq 2$. In [10], Mophou proved the existence and uniqueness results of a mild solution to impulsive fractional semilinear differential equations. Anguraj and Karthikeyan [3] proved Existence for impulsive neutral integrodifferential inclusions with nonlocal initial conditions via fractional operators. Benchohra and Seba [6] studied the existence of fractional impulsive differential equations in Banach spaces while Balachandran and Kiruthika [5] discussed the existence of nonlocal cauchy problem for semilinear fractional evoluation equations. Balachandran and Trujillo [4] investigated the nonlocal Cauchy problem for nonlinear fractional integrodifferential equations in Banach spaces.

In this paper, we consider the following fractional impulsive neutral integrodifferential systems with infinite delay

$$
\left\{\begin{array}{lc}
D_{t}^{q}\left(x(t)-u\left(t, x_{t}\right)\right)=A(t, x)\left(x(t)-u\left(t, x_{t}\right)\right)+f\left(t, x_{t}, \int_{0}^{t} h\left(t, s, x_{s}\right) d s\right), & t \in J=[0, b], \quad t \neq t_{k} \\
\left.\Delta x\right|_{t=t_{k}}=I_{k}\left(x\left(t_{k}^{-}\right)\right), & t=t_{k}, k=1,2, \ldots, n \\
x(0)+g(x)=\phi, \quad \phi \in B_{\vartheta} &
\end{array}\right.
$$

where $0<q<1$ and the state $\mathrm{x}($.$) belongs to Banach space \mathrm{X}$ endowed with the norm $\|$.$\| . Operator A$ generates a strongly continuous bounded linear operator on a Banach space X. $D_{t}^{q}$ is the Caputo fractional derivative. $\mathrm{u}$ and $\mathrm{f}$ are two given continuous functions, $I_{k}: X \rightarrow X, \Delta x\left(t_{k}\right)=x\left(t_{k}^{+}\right)-x\left(t_{k}^{-}\right)$with $x\left(t_{k}^{+}\right)=$ $\lim _{h \rightarrow 0^{+}} x\left(t_{k}+h\right), x\left(t_{k}^{-}\right)=\lim _{h \rightarrow 0^{-}} x\left(t_{k}+h\right), k=1,2,3, \ldots . . n, 0=t_{0}<t_{1}<t_{2}<\ldots<t_{n}<t_{n+1}=b$. Let $x_{t}($.$) denote x_{t}(\theta)=x(t+\theta), \theta \in(-\infty, 0]$.

The rest of this papper is organized as follows. In Section 2, some preliminaries are presented. In Section 3 , we study the existence and the uniqueness of solutions for the impulsive fractional system 1.1. In Section 4 , an example.

\section{Preliminaries}

In this section, we shall introduce some basic definitions, notations, lemmas and proposition which are used throughout this paper.

Assume that $\vartheta:(-\infty, 0] \rightarrow(0,+\infty)$ is a continuous function satisfyi $\ell=\int_{-\infty}^{0} \vartheta(t) d t<+\infty$. The Banach space $\left(B_{\vartheta},\|.\|_{B_{\vartheta}}\right)$ induced by the function $\vartheta$ is defined as follows

$$
B_{\vartheta}=\left\{\begin{array}{l}
\varphi:(-\infty, 0] \longrightarrow X: \text { for any } c>0, \varphi(\theta) \text { is a bounded and } \\
\text { measurable function on }[-c, 0] \text { and } \int_{-\infty}^{0} \vartheta(t) \sup _{t \leq \theta \leq 0}|\varphi(\theta)| d t<+\infty
\end{array}\right.
$$

endowed with the norm $\|\varphi\|_{B_{\vartheta}}=\int_{-\infty}^{0} \vartheta(s) \sup _{s \leq \theta \leq 0}|\varphi(\theta)| d s$.

Let us define the space

$$
B_{\vartheta^{\prime}}=\left\{\begin{array}{l}
\varphi:(-\infty, b] \rightarrow X: \varphi_{k} \in C\left(J_{k}, X\right), k=0,1,2, \ldots n \text { and there exist } \\
\varphi\left(t_{k}^{-}\right) \text {and } \varphi\left(t_{k}^{+}\right) \text {with } \varphi\left(t_{k}\right)=\varphi\left(t_{k}^{-}\right), \varphi_{0}=\varphi(0)+g(\varphi)=\phi \in B_{\vartheta}
\end{array}\right.
$$

where $\varphi_{k}$ is the restriction of $\varphi$ to $J_{k}, J_{0}=\left[0, t_{1}\right], J_{k}=\left(t_{k}, t_{k+1}\right], k=1,2, \ldots . n$. Denote by $\|\cdot\|_{B_{\vartheta^{\prime}}}$, a seminorm in the space $B_{\vartheta}^{\prime}$, which is defined by

$\|\varphi\|_{B_{\vartheta^{\prime}}}=\|\varphi\|_{B_{\vartheta}}+\max \left\|\varphi_{k}\right\|_{J_{k}}, k=1,2, \ldots n$ where $\left\|\varphi_{k}\right\|_{J_{k}}=\sup _{s \in J_{k}}\left\|\varphi_{k}(s)\right\|$. 
Definition 2.1. A function $x:(-\infty, b] \rightarrow X$ is said to be a solution of system 1.1 if $x(0)+g(x)=\phi \in B_{\vartheta^{\prime}}$, the impulsive condition $\left.\Delta x\right|_{t=t_{k}}=I_{k}\left(x\left(t_{k}^{-}\right)\right), k=1,2, \ldots, n$ is verified, the restriction of $\mathrm{x}($.$) to the interval$ $J_{k}(k=0,1,2, \ldots n)$ is continuous and the following integral equation holds for $t \in J$,

$$
x(t)=\left\{\begin{array}{l}
{[\phi(0)-g(x)-u(0, \phi)]+u\left(t, x_{t}\right)} \\
+\frac{1}{\Gamma(q)} \sum_{0<t_{k}<t} \int_{t_{k-1}}^{t_{k}}\left(t_{k}-s\right)^{q-1} A(s, x) x(s) d s \\
+\frac{1}{\Gamma(q)} \int_{t_{k}}^{t}(t-s)^{q-1} A(s, x) x(s) d s \\
-\frac{1}{\Gamma(q)} \sum_{0<t_{k}<t} \int_{t_{k-1}}^{t_{k}}\left(t_{k}-s\right)^{q-1} A(s, x) u\left(s, x_{s}\right) d s \\
-\frac{1}{\Gamma(q)} \int_{t_{k}}^{t}(t-s)^{q-1} A(s, x) u\left(s, x_{s}\right) d s \\
+\frac{1}{\Gamma(q)} \sum_{0<t_{k}<t} \int_{t_{k-1}}^{t_{k}}\left(t_{k}-s\right)^{q-1} f\left(s, x_{s}, \int_{0}^{s} h\left(s, \tau, x_{\tau}\right) d \tau\right) d s \\
+\frac{1}{\Gamma(q)} \int_{t_{k}}^{t}(t-s)^{q-1} f\left(s, x_{s}, \int_{0}^{s} h\left(s, \tau, x_{\tau}\right) d \tau\right) d s \\
+\sum_{0<t_{k}<t} I_{k}\left(x\left(t_{k}^{-}\right)\right)
\end{array}\right.
$$

Definition 2.2. The Riemann - Liouville fractional integral operator of order $q \geq 0$ of function $f \in L^{1}\left(R^{+}\right)$ is defined as

$$
I_{0^{+}}^{q} f(t)=\frac{1}{\Gamma(q)} \int_{0}^{t}(t-s)^{q-1} f(s) d s, \quad t>0
$$

where $\Gamma($.$) is the Euler gamma function.$

Definition 2.3. The Caputo fractional derivative of order $q \geq 0, n-1<q<n$, is defined as

$$
D_{0^{+}}^{q} f(t)=\frac{1}{\Gamma(n-q)} \int_{0}^{t}(t-s)^{(n-q-1)} f^{(n)}(s) d s, \quad t>0
$$

where the function $\mathrm{f}(\mathrm{t})$ has absolutely continuous derivatives up to order (n-1).

If $0<q<1$, then

$$
D_{0^{+}}^{q} f(t)=\frac{1}{\Gamma(1-q)} \int_{0}^{t}(t-s)^{(-q)} f^{(1)}(s) d s
$$

where $f^{(1)}(s)=D f(s)=\frac{d f(s)}{d s}$ and $\mathrm{f}$ is an abstract function with values in $\mathrm{X}$.

We shall state some properties of the operators $I_{0^{+}}^{\alpha}$ and $D_{0^{+}}^{\alpha}$.

Lemma 2.4. . For $\alpha, \beta>o$ and $f$ as a suitable function, we have

(i) $I^{\alpha} I^{\beta} f(t)=I^{\alpha+\beta} f(t)$

(ii) $I^{\alpha} I^{\beta} f(t)=I^{\beta} I^{\alpha} f(t)$

(iii) $I^{\alpha}(f(t)+g(t))=I^{\alpha} f(t)+I^{\alpha} g(t)$

(iv) $I^{\alpha c} D^{\alpha} f(t)=f(t)-f(0), 0<\alpha<1$

(v) ${ }^{c} D^{\alpha} I^{\alpha} f(t)=f(t)$

(vi) ${ }^{c} D^{\alpha} f(t)=I^{(1-\alpha)} D f(t)=I^{(1-\alpha)} f^{\prime}(t), 0<\alpha<1, D=\frac{d}{d t}$

(vii) ${ }^{c} D^{\alpha c} D^{\beta} f(t) \neq{ }^{c} D^{(\alpha+\beta)} f(t)$ 
(viii) ${ }^{c} D^{\alpha c} D^{\beta} f(t) \neq{ }^{c} D^{\beta}{ }^{c} D^{\alpha} f(t)$

Zhang and Xiyue Huang [18] proved the existence and uniqueness of mild solutions for impulsive fractional equations with nonlocal conditions and infinite delay, in which $\mathrm{A}$ is a infinitesimal generator of strongly continuous semigroup. But in [4], Balachandran and Trujillo observed that both the R - L and the Caputo fractional differential operators do not possess neither semigroup nor commutative properties, which are inherent to the derivatives on integer order.

Theorem 2.5. ( $[17\rceil)$

Let $B$ be a convex, bounded and closed subset of a Banach space $X$ and $N: B \rightarrow B$ be a condensing map. Then $N$ has a fixed point in $B$.

Lemma 2.6. ([9])

Assume that $x \in B_{\vartheta^{\prime}}$ then, for $t \in J, x_{t} \in B_{\vartheta}$. Moreover

$$
\ell|| x(t)\|\leq\| x_{t}\left\|_{B_{\vartheta}} \leq\right\| \phi\left\|_{B_{\vartheta}}+\ell \sup _{s \in[0, t]}\right\| x(s) \| .
$$

\section{Main results}

For $\phi \in B_{\vartheta}$, we define $\hat{\phi}$ by

$$
\hat{\phi}(t)=\left\{\begin{array}{l}
\phi(t), t \in(-\infty, 0] \\
\phi(0), t \in J
\end{array}\right.
$$

then $\hat{\phi} \in B_{\vartheta^{\prime}}$.

Let $x(t)=y(t)+\hat{\phi}(t),-\infty<t<b$.

It is evident that $\mathrm{y}$ satisfies $y_{0}=0, t \in(-\infty, 0]$, and

$$
\begin{aligned}
x(t)= & {[-g(y+\hat{\phi})-u(0, \phi)]+u\left(t, y_{t}+\hat{\phi}_{t}\right) } \\
& +\frac{1}{\Gamma(q)} \sum_{0<t_{k}<t} \int_{t_{k-1}}^{t_{k}}\left(t_{k}-s\right)^{q-1} A(s, y+\hat{\phi})(y+\hat{\phi})(s) d s \\
& +\frac{1}{\Gamma(q)} \int_{t_{k}}^{t}(t-s)^{q-1} A(s, y+\hat{\phi})(y+\hat{\phi})(s) d s \\
& -\frac{1}{\Gamma(q)} \sum_{0<t_{k}<t} \int_{t_{k-1}}^{t_{k}}\left(t_{k}-s\right)^{q-1} A(s, y+\hat{\phi}) u\left(s, y_{s}+\hat{\phi}_{s}\right) d s \\
& -\frac{1}{\Gamma(q)} \int_{t_{k}}^{t}(t-s)^{q-1} A(s, y+\hat{\phi}) u\left(s, y_{s}+\hat{\phi}_{s}\right) d s \\
& +\frac{1}{\Gamma(q)} \sum_{0<t_{k}<t} \int_{t_{k-1}}^{t_{k}}\left(t_{k}-s\right)^{q-1} f\left(s, y_{s}+\hat{\phi}_{s}, \int_{0}^{s} h\left(s, \tau, y_{\tau}+\hat{\phi}_{\tau}\right) d \tau\right) d s \\
& +\frac{1}{\Gamma(q)} \int_{t_{k}}^{t}(t-s)^{q-1} f\left(s, y_{s}+\hat{\phi}_{s}, \int_{0}^{s} h\left(s, \tau, y_{\tau}+\hat{\phi}_{\tau}\right) d \tau\right) d s \\
& +\sum_{0<t_{k}<t} I_{k}\left(y\left(t_{k}^{-}\right)+\hat{\phi}\left(t_{k}^{-}\right)\right),
\end{aligned}
$$

if and only if $\mathrm{x}$ satisfies $x(t)=\phi(t), t \in(-\infty, 0]$ and $\mathrm{x}$ satisfies equation 2.1.

For brevity let us take

$$
H\left(x_{s}\right)=\int_{0}^{s} h\left(s, \tau, x_{\tau}\right) d \tau .
$$

We assume the following conditions to prove the existence of solution of the equation 1.1 . 
$\left(H_{1}\right) A: J \times B_{\vartheta} \rightarrow B(X)$ is a continuous bounded linear operator and there exists a constant $M>0$, such that $\|A(t, x)-A(t, y)\| \leq M\|x-y\|_{B_{\vartheta}}$, for all $x, y \in B_{\vartheta}$.

$\left(H_{2}\right)$ The function $u: J \times B_{\vartheta} \rightarrow X$, and there exist two positive constants $\lambda_{1}$ and $\lambda_{2}$ such that the function satisfies the Lipschitz condition $\left\|u\left(s, x_{t}\right)-u\left(s, y_{t}\right)\right\| \leq \lambda_{1}\left(\left\|x_{t}-y_{t}\right\|_{B_{\vartheta^{\prime}}}\right), \quad \lambda_{2}=\sup _{t \in J}\|u(t, 0)\|$.

( $\left.H_{3}\right) f: J \times B_{\vartheta} \times X \rightarrow X$, and there exist two positive constants $K_{1}, K_{2}$ such that $\left\|f\left(t, \phi_{1}, y_{1}\right)-f\left(t, \phi_{2}, y_{2}\right)\right\| \leq K_{1}\left(\left\|\phi_{1}-\phi_{2}\right\|_{B_{\vartheta}}+\| y_{1}-y_{2}||\right)$, $K_{2}=\sup _{t \in J}|| f(t, 0,0) \|$.

$\left(H_{4}\right) h: \Delta \times B_{\vartheta} \rightarrow X$, where $\Delta=\{(t, s): 0 \leq s \leq b\}$, equipped with positive constants $P_{1}, P_{2}$ satisfying $\left\|h\left(t, s, \phi_{1}\right)-h\left(t, s, \phi_{2}\right)\right\| \leq P_{1}\left(\left\|\phi_{1}-\phi_{2}\right\|_{B_{\vartheta^{\prime}}}\right), \quad P_{2}=\sup _{(t, s)}\|h(t, s, 0)\|$.

$\left(H_{5}\right) I_{k}: X \rightarrow X$ are continuous, and there exists a constant $\mu>0$ such that $\left\|I_{k}(x)-I_{k}(y)\right\| \leq \mu\|x-y\|, \quad k=1,2,3, \ldots . . n$.

$\left(H_{6}\right) g: B_{\vartheta^{\prime}} \rightarrow X$ is continuous and there exist some positive constant $\delta_{1}, \delta_{2}$ such that $\|g(x)-g(y)\| \leq \delta_{1}\|x-y\|_{B_{\vartheta}^{\prime}}$ and $\|g(x)\| \leq \delta_{1}\|x\|_{B_{\vartheta}^{\prime}}+\delta_{2}$.

$\left(H_{7}\right)\left[\delta_{1} L+\delta_{2}+\lambda_{1}\left(L_{1}+\|\phi\|_{B_{\vartheta}}\right)+2 \lambda_{2}+T+\mu n\left(r+\ell^{-1}\|\phi\|_{B_{\vartheta}}+|\phi(0)|\right)\right] \leq r$ where $T=\frac{b^{q}(n+1)}{\Gamma(q+1)}\left[(M L+K)\left(L+\lambda_{1} L_{1}+\lambda_{2}\right)+K_{1} L_{1}+K_{1} b\left(P_{1} L_{1}+P_{2}\right)+K_{2}\right]$ and $L=r+\|\phi\|_{B_{\vartheta}}+|\phi(0)|, L_{1}=\ell(r+|\phi(0)|)+\|\phi\|_{B_{\vartheta}}$.

Theorem 3.1. Suppose that conditions $\left(H_{1}\right)-\left(H_{7}\right)$ are satisfied with $\delta_{1}+\lambda_{1} \ell<1$ then system 1.1 has a solution.

Proof 1. Define $\Theta: B_{\vartheta^{\prime}} \rightarrow B_{\vartheta^{\prime}}$ by

$$
\begin{array}{rlr}
\Theta y(t)= & 0, & \\
\Theta y(t)= & -g(y+\hat{\phi})-u(0, \phi)]+u\left(t, y_{t}+\hat{\phi}_{t}\right) & t \in(-\infty, 0] \\
& +\frac{1}{\Gamma(q)} \sum_{0<t_{k}<t} \int_{t_{k-1}}^{t_{k}}\left(t_{k}-s\right)^{q-1} A(s, y+\hat{\phi})(y+\hat{\phi})(s) d s & \\
& +\frac{1}{\Gamma(q)} \int_{t_{k}}^{t}(t-s)^{q-1} A(s, y+\hat{\phi})(y+\hat{\phi})(s) d s \\
& -\frac{1}{\Gamma(q)} \sum_{0<t_{k}<t} \int_{t_{k-1}}^{t_{k}}\left(t_{k}-s\right)^{q-1} A(s, y+\hat{\phi}) u\left(s, y_{s}+\hat{\phi}_{s}\right) d s \\
& -\frac{1}{\Gamma(q)} \int_{t_{k}}^{t}(t-s)^{q-1} A(s, y+\hat{\phi}) u\left(s, y_{s}+\hat{\phi}_{s}\right) d s \\
& +\frac{1}{\Gamma(q)} \sum_{0<t_{k}<t} \int_{t_{k-1}}^{t_{k}}\left(t_{k}-s\right)^{q-1} f\left(s, y_{s}+\hat{\phi}_{s}, H\left(y_{s}+\hat{\phi}_{s}\right)\right) d s \\
& +\frac{1}{\Gamma(q)} \int_{t_{k}}^{t}(t-s)^{q-1} f\left(s, y_{s}+\hat{\phi}_{s}, H\left(y_{s}+\hat{\phi}_{s}\right)\right) d s \\
& +\sum_{0<t_{k}<t} I_{k}\left(y\left(t_{k}^{-}\right)+\hat{\phi}\left(t_{k}^{-}\right)\right),
\end{array}
$$

Clearly, $\mathrm{y}$ is a fixed point of $\Theta$ then $y+\hat{\phi}$ is a solution of the system 1.1. We shall show that $\Theta$ satisfies the hypotheses of Theorem 2.5 . 
Define the Banach space $\left(B_{\vartheta^{\prime \prime}},\|\cdot\|_{B_{\vartheta^{\prime}}}\right)$ induced by $B_{\vartheta^{\prime}}$,

$B_{\vartheta^{\prime \prime}}=\left\{y \in B_{\vartheta^{\prime}}: y_{0}=0 \in B_{\vartheta}\right\}$ with norm $\|y\|_{B_{\vartheta^{\prime}}}=\sup \{|y(s)|: s \in[0, b]\}$.

Set $B_{r}=\left\{y \in B_{\vartheta^{\prime \prime}}:\|y\|_{B_{\vartheta^{\prime}}} \leq r\right\}$ for some $r>0$ then $B_{r}$, for each $\mathrm{r}$, is bounded, closed convex subset of X. For any $y \in B_{r}$, by Lemma 2.6, we have

$$
\begin{aligned}
\left\|y_{t}+\hat{\phi}_{t}\right\|_{B_{\vartheta}} & \leq\|\phi\|_{B_{\vartheta}}+\ell[r+|\phi(0)|], \\
\|y+\hat{\phi} \mid\|_{B_{\vartheta^{\prime}}} & \leq r+\|\phi\|_{B_{\vartheta}}+|\phi(0)|, \\
\sup _{t \in J}|y(t)+\hat{\phi}(t)| & \leq r+\ell^{-1}|| \phi \|_{B_{\vartheta}}+|\phi(0)| .
\end{aligned}
$$

Now we proceed in Two steps.

Step I:We claim that there exists a positive integer $r \in N$ such that $\Theta\left(B_{r}\right) \subset B_{r}$.

There exists a positive number $\mathrm{r}$ such that $B_{r}$ is clearly a closed bounded convex set in $B_{\vartheta^{\prime}}$.

For each positive integer $\mathrm{r}$, there exist $y_{r} \in B_{r}$ and $t(r) \in(-\infty, b]$ such that

$$
\begin{aligned}
\left\|\Theta\left(y^{r}\right)(t(r))\right\| \leq & \left\|\left[-g\left(y^{r}+\hat{\phi}\right)-u(0, \phi)\right]\right\|+\left\|u\left(t(r), y_{t(r)}+\hat{\phi}_{t(r)}\right)\right\| \\
& +\frac{1}{\Gamma(q)} \sum_{0<t_{k}<t(r)} \int_{t_{k-1}}^{t_{k}}\left(t_{k}-s\right)^{q-1}\left\|A\left(s, y^{r}+\hat{\phi}\right)\left(y^{r}+\hat{\phi}\right)(s)\right\| d s \\
& +\frac{1}{\Gamma(q)} \int_{t_{k}}^{t(r)}(t(r)-s)^{q-1}\left\|A\left(s, y^{r}+\hat{\phi}\right)\left(y^{r}+\hat{\phi}\right)(s)\right\| d s \\
& -\frac{1}{\Gamma(q)} \sum_{0<t_{k}<t(r)} \int_{t_{k-1}}^{t_{k}}\left(t_{k}-s\right)^{q-1}\left\|A\left(s, y^{r}+\hat{\phi}\right) u\left(s, y_{s}^{r}+\hat{\phi}_{s}\right)\right\| d s \\
& -\frac{1}{\Gamma(q)} \int_{t_{k}}^{t(r)}(t(r)-s)^{q-1}\left\|A\left(s, y^{r}+\hat{\phi}\right) u\left(s, y_{s}^{r}+\hat{\phi}_{s}\right)\right\| d s \\
& +\frac{1}{\Gamma(q)} \sum_{0<t_{k}<t(r)} \int_{t_{k-1}}^{t_{k}}\left(t_{k}-s\right)^{q-1}\left\|f\left(s, y_{s}^{r}+\hat{\phi}_{s}, H\left(y_{s}^{r}+\hat{\phi}_{s}\right)\right)\right\| d s \\
& +\frac{1}{\Gamma(q)} \int_{t_{k}}^{t(r)}(t(r)-s)^{q-1}\left\|f\left(s, y_{s}^{r}+\hat{\phi}_{s}, H\left(y_{s}^{r}+\hat{\phi}_{s}\right)\right)\right\| d s \\
& +\sum_{0<t_{k}<t(r)}\left\|I_{k}\left(y^{r}\left(t_{k}^{-}\right)+\hat{\phi}\left(t_{k}^{-}\right)\right)\right\| \\
\leq & \delta_{1} L+\delta_{2}+\lambda_{1}\left(L_{1}+\|\phi\|_{B_{\vartheta}}\right)+2 \lambda_{2} \\
& +\frac{b^{q}(n+1)}{\Gamma(q+1)}\left[(M L+K)\left(L+\lambda_{1} L_{1}+\lambda_{2}\right)+K_{1} L_{1}\right. \\
& \left.+K_{1} b\left(P_{1} L_{1}+P_{2}\right)+K_{2}\right]+\mu n\left(r+\ell^{-1}\|\phi\|_{B_{\vartheta}}+\mid \phi(0) \|\right) \\
\leq & r
\end{aligned}
$$

where $L=r+|| \phi\left\|_{B_{\vartheta}}+|\phi(0)|, \quad L_{1}=\ell(r+|\phi(0)|)+\right\| \phi \|_{B_{\vartheta}}$.

Using $\left(H_{7}\right)$, for some positive integer $\mathrm{r}, \Theta\left(B_{r}\right) \subset B_{r}$.

Step II:Now we claim that the operator $\Theta=\Theta_{1}+\Theta_{2}$ is condensing, that is $\Theta_{1}$ is a contraction and $\Theta_{2}$ is compact.

The operators $\Theta_{1}$ and $\Theta_{2}$ are defined on $B_{r}$ respectively by,

$$
\left(\Theta_{1} y\right)(t)=\left\{\begin{array}{cc}
0, & t \in(-\infty, 0] \\
{[-g(y+\hat{\phi})-u(0, \phi)]+u\left(t, y_{t}+\hat{\phi}_{t}\right),} & t \in J .
\end{array}\right.
$$




$$
\left(\Theta_{2} y\right)(t)=\left\{\begin{array}{rlrl}
0 & , & t \in(-\infty, 0] \\
\frac{1}{\Gamma(q)} & \sum_{0<t_{k}<t} \int_{t_{k-1}}^{t_{k}}\left(t_{k}-s\right)^{q-1} A(s, y+\hat{\phi})(y+\hat{\phi})(s) d s & \\
& +\frac{1}{\Gamma(q)} \int_{t_{k}}^{t}(t-s)^{q-1} A(s, y+\hat{\phi})(y+\hat{\phi})(s) d s \\
& -\frac{1}{\Gamma(q)} \sum_{0<t_{k}<t}^{t_{t_{k-1}}}\left(t_{k}-s\right)^{q-1} A(s, y+\hat{\phi}) u\left(s, y_{s}+\hat{\phi}_{s}\right) d s & \\
& -\frac{1}{\Gamma(q)} \int_{t_{k}}^{t}(t-s)^{q-1} A(s, y+\hat{\phi}) u\left(s, y_{s}+\hat{\phi}_{s}\right) d s & \\
& +\frac{1}{\Gamma(q)} \sum_{0<t_{k}<t} \int_{t_{k-1}}^{t_{k}}\left(t_{k}-s\right)^{q-1} f\left(s, y_{s}+\hat{\phi}_{s}, H\left(y_{s}+\hat{\phi}_{s}\right)\right) d s & t \in J . \\
& +\frac{1}{\Gamma(q)} \int_{t_{k}}^{t}(t-s)^{q-1} f\left(s, y_{s}+\hat{\phi}_{s}, H\left(y_{s}+\hat{\phi}_{s}\right)\right) d s & \\
& +\sum_{0<t_{k}<t} I_{k}\left(y\left(t_{k}^{-}\right)+\hat{\phi}\left(t_{k}^{-}\right)\right),
\end{array}\right.
$$

We take $y_{1}, y_{2} \in B_{r}$ arbitrarily.

By $\left(H_{2}\right)$ and $\left(H_{6}\right)$, we have

$$
\left\|\left(\Theta_{1} y_{1}\right)(t)-\left(\Theta_{1} y_{2}\right)(t)\right\| \leq \delta_{1}\left\|y_{1}-y_{2}\right\|_{B_{\vartheta^{\prime}}}+\lambda_{1} \ell\left\|y_{1}-y_{2}\right\|_{B_{\vartheta^{\prime}}}
$$

since $\left\|y_{t}\right\|_{B_{\vartheta}} \leq \ell\|y\|_{B_{\vartheta^{\prime}}}$

$$
\begin{aligned}
\left\|\left(\Theta_{1} y_{1}\right)(t)-\left(\Theta_{1} y_{2}\right)(t)\right\| & \leq\left(\delta_{1}+\lambda_{1} \ell\right)\left\|y_{1}-y_{2}\right\|_{B_{\vartheta^{\prime}}} \\
& \leq\left\|y_{1}-y_{2}\right\|_{B_{\vartheta^{\prime}}}
\end{aligned}
$$

since $\delta_{1}+\lambda_{1} \ell<1$. Therefore $\Theta_{1}$ is a contraction.

Next, we prove that $\Theta_{2}$ is continuous on $B_{r}$.

Let $\left\{y_{m}\right\}_{k=0}^{\infty} \subseteq B_{r}$, with $y_{m} \rightarrow y$ in $B_{r}$. By (H1), (H3), (H4) and (H5) we have

$$
\begin{aligned}
\left\|\left(\Theta_{2} y\right)(t)-\left(\Theta_{2} y_{m}\right)(t)\right\| \leq & \frac{1}{\Gamma(q)} \sum_{0<t_{k}<t} \int_{t_{k-1}}^{t_{k}}\left(t_{k}-s\right)^{q-1} \| A(s, y+\hat{\phi})(y+\hat{\phi})(s) \\
& -A\left(s, y_{m}+\hat{\phi}\right)\left(y_{m}+\hat{\phi}\right)(s) \| d s \\
& +\frac{1}{\Gamma(q)} \int_{t_{k}}^{t}(t-s)^{q-1} \| A(s, y+\hat{\phi})(y+\hat{\phi})(s) \\
& -A\left(s, y_{m}+\hat{\phi}\right)\left(y_{m}+\hat{\phi}\right)(s) \| d s \\
& +\frac{1}{\Gamma(q)} \sum_{0<t_{k}<t} \int_{t_{k-1}}^{t_{k}}\left(t_{k}-s\right)^{q-1} \| A(s, y+\hat{\phi}) u\left(s, y_{s}+\hat{\phi}_{s}\right) \\
& -A\left(s, y_{m}+\hat{\phi}\right) u\left(s, y_{m s}+\hat{\phi}_{s}\right) \| d s \\
& +\frac{1}{\Gamma(q)} \int_{t_{k}}^{t}(t-s)^{q-1} \| A(s, y+\hat{\phi}) u\left(s, y_{s}+\hat{\phi}_{s}\right) \\
& -A\left(s, y_{m}+\hat{\phi}\right) u\left(s, y_{m s}+\hat{\phi}_{s}\right) \| d s \\
& +\frac{1}{\Gamma(q)} \sum_{0<t_{k}<t} \int_{t_{k-1}}^{t_{k}}\left(t_{k}-s\right)^{q-1} \| f\left(s, y_{s}+\hat{\phi}_{s}, H\left(y_{s}+\hat{\phi}_{s}\right)\right) \\
& -f\left(s, y_{m s}+\hat{\phi}_{s}, H\left(y_{m s}+\hat{\phi}_{s}\right)\right) \| d s \\
& +\frac{1}{\Gamma(q)} \int_{t_{k}}^{t}(t-s)^{q-1} \| f\left(s, y_{s}+\hat{\phi}_{s}, H\left(y_{s}+\hat{\phi}_{s}\right)\right) \\
& -f\left(s, y_{m s}+\hat{\phi}_{s}, H\left(y_{m s}+\hat{\phi}_{s}\right)\right) \| d s \\
& +\sum_{0<t_{k}<t}\left\|I_{k}\left(y\left(t_{k}^{-}\right)+\hat{\phi}\left(t_{k}^{-}\right)\right)-I_{k}\left(y_{m}\left(t_{k}^{-}\right)+\hat{\phi}\left(t_{k}^{-}\right)\right)\right\|
\end{aligned}
$$




$$
\begin{aligned}
\leq & \frac{(n+1) b^{q}}{\Gamma(q+1)}\left[\left(M\left[r+\|\phi\|_{B_{\vartheta}}+|\phi(0)|\right]+K\right)\left(1+\ell \lambda_{1}\right)\right. \\
& +M\left(r+|\phi(0)|+\|\phi\|_{B_{\vartheta}}+\lambda_{1}[\ell(r+|\phi(0)|)\right. \\
& \left.\left.\left.+\|\phi\|_{B_{\vartheta}}\right]+\lambda_{2}\right)+K_{1} \ell\left(1+\frac{P_{1} b}{(q+1)}\right)\right]|| y-y_{m} \|_{B_{\vartheta^{\prime}}} \\
& +\sum_{0<t_{k}<t}\left\|I_{k}\left(y\left(t_{k}^{-}\right)+\hat{\phi}\left(t_{k}^{-}\right)\right)-I_{k}\left(y_{m}\left(t_{k}^{-}\right)+\hat{\phi}\left(t_{k}^{-}\right)\right)\right\| .
\end{aligned}
$$

$\left\|\left(\Theta_{2} y\right)(t)-\left(\Theta_{2} y_{m}\right)(t)\right\| \rightarrow 0$ as $m \rightarrow \infty$

Thus, $\Theta_{2}$ is continuous.

Next, we prove that $\left\{\Theta_{2} y: y \in B_{r}\right\}$ is a family of equicontinuous functions.

Let $0<t_{1}<t_{2} \leq b$. Then

$$
\left\|\left(\Theta_{2} y\right)\left(t_{2}\right)-\left(\Theta_{2} y\right)\left(t_{1}\right)\right\| \leq I_{1}+I_{2}+I_{3}+I_{4}+I_{5}+I_{6}+I_{7}
$$

where

$$
\begin{aligned}
I_{1}= & \frac{1}{\Gamma(q)}\left[\| \sum_{0<t_{k}<t_{2}} \int_{t_{k-1}}^{t_{k}}\left(t_{k}-s\right)^{q-1} A(s, y+\hat{\phi})(y+\hat{\phi})(s) d s\right. \\
& \left.-\sum_{0<t_{k}<t_{1}} \int_{t_{k-1}}^{t_{k}}\left(t_{k}-s\right)^{q-1} A(s, y+\hat{\phi})(y+\hat{\phi})(s) d s \|\right], \\
I_{2}= & \frac{1}{\Gamma(q)}\left[\left\|\int_{t_{k}}^{t_{2}}\left(t_{2}-s\right)^{q-1} A(s, y+\hat{\phi})(y+\hat{\phi})(s) d s-\int_{t_{k}}^{t_{1}}\left(t_{1}-s\right)^{q-1} A(s, y+\hat{\phi})(y+\hat{\phi})(s) d s\right\|\right], \\
I_{3}= & \frac{1}{\Gamma(q)}\left[\| \sum_{0<t_{k}<t_{2}} \int_{t_{k-1}}^{t_{k}}\left(t_{k}-s\right)^{q-1} A(s, y+\hat{\phi}) u\left(s, y_{s}+\hat{\phi}_{s}\right) d s\right. \\
& \left.-\sum_{0<t_{k}<t_{1}} \int_{t_{k-1}}^{t_{k}}\left(t_{k}-s\right)^{q-1} A(s, y+\hat{\phi}) u\left(s, y_{s}+\hat{\phi}_{s}\right) d s \|\right], \\
I_{4}= & \frac{1}{\Gamma(q)}\left[\left\|\int_{t_{k}}^{t_{2}}\left(t_{2}-s\right)^{q-1} A(s, y+\hat{\phi}) u\left(s, y_{s}+\hat{\phi}_{s}\right) d s-\int_{t_{k}}^{t_{1}}\left(t_{1}-s\right)^{q-1} A(s, y+\hat{\phi}) u\left(s, y_{s}+\hat{\phi}_{s}\right) d s\right\|\right], \\
I_{5}= & \frac{1}{\Gamma(q)}\left[\| \sum_{0<t_{k}<t_{2}} \int_{t_{k-1}}^{t_{k}}\left(t_{k}-s\right)^{q-1} f\left(s, y_{s}+\hat{\phi}_{s}, H\left(y_{s}+\hat{\phi}_{s}\right)\right) d s\right. \\
I_{6}= & \frac{1}{\Gamma(q)}\left[\left\|\int_{t_{k}}^{t_{2}}\left(t_{2}-s\right)^{q-1} f\left(s, y_{s}+\hat{\phi}_{s}, H\left(y_{s}+\hat{\phi}_{s}\right)\right) d s-\int_{t_{k}}^{t_{1}}\left(t_{1}-s\right)^{q-1} f\left(s, y s+\hat{\phi}_{s}, H\left(y_{s}+\hat{\phi}_{s}\right)\right) d s\right\|\right], \\
I_{7}= & \left\|\sum_{0<t_{k}<t_{2}} I_{k}\left(y\left(t_{k}^{-}\right)+\hat{\phi}\left(t_{k}^{-}\right)\right)-\sum_{0<t_{k}<t_{1}} I_{k}\left(y\left(t_{k}^{-}\right)+\hat{\phi}\left(t_{k}^{-}\right)\right)\right\| .
\end{aligned}
$$

Clearly $I_{1}, I_{2}, I_{3}, I_{4}, I_{5}, I_{6}, I_{7}$ tends to 0 when $t_{1} \rightarrow t_{2}$.

Therefore, we conclude that $\lim _{t_{1} \rightarrow t_{2}} I_{i}=0, i=1,2,3,4,5,6,7$. Hence $\Theta_{2} B_{r}$ is equicontinuous.

Next, we claim that $\Theta_{2} B_{r}$ is precompact. 
Let $0<t \leq b$ be fixed and let $\epsilon$ be a real number satisfying $0<\epsilon<b$. For $y \in B_{r}$, we define

$$
\begin{aligned}
\left(\Theta_{2}^{\epsilon} y\right)(t)= & \frac{1}{\Gamma(q)} \sum_{0<t_{k}<t} \int_{t_{k-1}}^{t_{k}}\left(t_{k}-s\right)^{q-1} A(s, y+\hat{\phi})(y+\hat{\phi})(s) d s \\
& +\frac{1}{\Gamma(q)} \int_{t_{k}}^{t-\epsilon}(t-s)^{q-1} A(s, y+\hat{\phi})(y+\hat{\phi})(s) d s \\
& -\frac{1}{\Gamma(q)} \sum_{0<t_{k}<t} \int_{t_{k-1}}^{t_{k}}\left(t_{k}-s\right)^{q-1} A(s, y+\hat{\phi}) u\left(s, y_{s}+\hat{\phi}_{s}\right) d s \\
& -\frac{1}{\Gamma(q)} \int_{t_{k}}^{t-\epsilon}(t-s)^{q-1} A(s, y+\hat{\phi}) u\left(s, y_{s}+\hat{\phi}_{s}\right) d s \\
& +\frac{1}{\Gamma(q)} \sum_{0<t_{k}<t} \int_{t_{k-1}}^{t_{k}}\left(t_{k}-s\right)^{q-1} f\left(s, y_{s}+\hat{\phi}_{s}, H\left(y_{s}+\hat{\phi}_{s}\right)\right) d s \\
& +\frac{1}{\Gamma(q)} \int_{t_{k}}^{t-\epsilon}(t-s)^{q-1} f\left(s, y_{s}+\hat{\phi}_{s}, H\left(y_{s}+\hat{\phi}_{s}\right)\right) d s \\
& +\sum_{0<t_{k}<t} I_{k}\left(y\left(t_{k}^{-}\right)+\hat{\phi}\left(t_{k}^{-}\right)\right)
\end{aligned}
$$

since the operator $\mathrm{A}(\mathrm{t}, \mathrm{x})$ is compact for $t>0$, for every $\epsilon$ sufficiently small, $0<\epsilon<b$,

$$
\begin{aligned}
\left\|\left(\Theta_{2} y\right)(t)-\left(\Theta_{2}^{\epsilon} y\right)(t)\right\|= & \| \frac{1}{\Gamma(q)} \int_{t-\epsilon}^{t}(t-s)^{q-1} A(s, y+\hat{\phi})(y+\hat{\phi})(s) d s \\
& -\frac{1}{\Gamma(q)} \int_{t-\epsilon}^{t}(t-s)^{q-1} A(s, y+\hat{\phi}) u\left(s, y_{s}+\hat{\phi}_{s}\right) d s \\
& +\frac{1}{\Gamma(q)} \int_{t-\epsilon}^{t}(t-s)^{q-1} f\left(s, y_{s}+\hat{\phi}_{s}, H\left(y_{s}+\hat{\phi}_{s}\right)\right) d s \| \\
\leq & \frac{1}{\Gamma(q+1)}\left[(M L+K)\left(\ell^{-1} L_{1}+\lambda_{1} L_{1}+\lambda_{2}\right)\right. \\
& \left.+\left(K_{1} L_{1}+K_{1} b\left(P_{1} L_{1}+P_{2}\right)+K_{2}\right)\right] \epsilon^{q} .
\end{aligned}
$$

Therefore, letting $\epsilon \rightarrow 0$, we see that there are relatively compact sets arbitrarily close to the set $\left\{\left(\Theta_{2} y\right)(t)\right.$ : $\left.y \in B_{r}\right\}$. Hence $\left\{\left(\Theta_{2} y\right)(t): y \in B_{r}\right\}$ is relatively compact in $B_{\vartheta^{\prime}}$.

As a consequence of the above steps and the Arzela-Ascoli Theorem, we can conclude that $\Theta_{2}$ is a compact operator. These arguments enable us to conclude that $\Theta=\Theta_{1}+\Theta_{2}$ is a condensing map on $B_{r}$ and Theorem 2.5 gives the conclusion that the system 1.1 has a solution.

\section{An Example}

Consider the following fractional integrodifferential equation with impulsive condition of the form

$$
\left\{\begin{array}{l}
D_{t}^{q}\left(x(t)-\frac{e^{-t} x}{\left(9+e^{t}\right)(1+x)}\right)=\frac{1}{4} \sin x(t)\left[x(t)-\frac{e^{-t} x}{\left(9+e^{t}\right)(1+x)}\right]+\frac{1}{(t+2)^{2}} \frac{|x|}{(1+|x|)}+\frac{1}{4} \int_{0}^{s} e^{\frac{-x_{s}}{3}} d s \\
\left.\Delta x\right|_{t=\frac{1}{2}}=\frac{\left|x\left(\frac{1}{2}\right)^{-}\right|}{3+\mid x\left(\frac{1}{2}\right)^{-1}} \\
x(0)+\frac{x}{5+x}=x_{0}
\end{array}\right.
$$

where $0<q \leq 1$ Take $J=[0,1], \mathrm{b}=1$

Let $A(t, x)=\frac{1}{4} \sin x(t), H\left(x_{s}\right)=\int_{0}^{s} e^{\frac{-x_{s}}{3}} d s$, 


$$
\begin{aligned}
& f\left(t, x, H\left(x_{s}\right)\right)=\frac{1}{(t+2)^{2}} \frac{|x|}{(1+|x|)}+\frac{1}{4} \int_{0}^{s} e^{\frac{-x_{s}}{3}} d s, \\
& u(t, x)=\frac{e^{-t} x}{\left(9+e^{t}\right)(1+x)}, \text { where } t \in J, x \in X=R .
\end{aligned}
$$

If $x, y \in X$ and $t \in J$ then we have

$$
\begin{aligned}
& \|A(t, x)-A(t, y)\| \leq \frac{1}{4}\|x-y\|,\left\|H\left(x_{s}\right)-H\left(y_{s}\right)\right\| \leq \frac{1}{3}\|x-y\|, \\
& \left\|f\left(t, x, H\left(x_{s}\right)\right)-f\left(t, y, H\left(y_{s}\right)\right)\right\| \leq \frac{1}{4}\left[\|x-y\|+\left\|H\left(x_{s}\right)-H\left(y_{s}\right)\right\|\right], \\
& \left\|I_{k}(x)-I_{k}(y)\right\| \leq \frac{1}{3}\|x-y\|,\left\|u\left(t, x_{t}\right)-u\left(t, y_{t}\right)\right\| \leq \frac{1}{10}\left\|x_{t}-y_{t}\right\|,\|g(x)-g(y)\| \leq \frac{1}{5}\|x-y\| .
\end{aligned}
$$

here $P_{1}=\frac{1}{3}, \quad K_{1}=\frac{1}{4}, \quad \mu=\frac{1}{3}, \quad \lambda_{1}=\frac{1}{10}, \quad \delta_{1}=\frac{1}{5}$.

Let $\vartheta(t)=e^{t}$, therefore $\ell=\int_{-\infty}^{0} \vartheta(t) d t=\int_{-\infty}^{0} e^{t} d t=1<+\infty$

hence $\delta_{1}+\lambda_{1} \ell=\frac{3}{10}<1$.

For some $q \in[0,1]$, all the hypotheses of the Theorem 2.5 are satisfied. Hence the problem 4.1 has a solution.

\section{Acknowledgements:}

The authors thank the referee for the valuable comments and suggestions.

\section{References}

[1] R. P. Agarwal, M. Benchohra, B. A. Slimani, Existence results for differential equations with fractional order and impulses, Memoirs on Differential Equations and Mathematical physics. 44 (2008), 1-21. 1

[2] A. Anguraj, P. Karthikeyan, G. M. N'Guéréata, Nonlocal cauchy problem for some fractional abstract integrodifferential equations in Banach spaces, Communications in Mathematical Analysis. 6 (1) (2009), 31-35. 1

[3] A. Anguraj, K. Karthikeyan, Y. K. Chang, Existence for impulsive neutral integrodifferential inclusions with nonlocal initial conditions via fractional operators, Nonlinear Analysis: Theory Methods and Applications. 71 (2009), 4377-4386. 1

[4] K. Balachandran, JJ. Trujillo, The non local cauchy problem for nonlinear fractional integrodifferential equations in Banach spaces. Nonlinear Analysis: Theory Methods and Applications. 7212 (2010), 4587-4593. 1, 2

[5] K. Balachandran, S. Kiruthika, JJ. Trujillo, Existence results for fractional impulsive integrodifferential equations in Banach spaces, Commun Nonlinear Sci Numer Simulat. 16 (4) (2011), 1970-1977. 1

[6] M. Benchohra, D. Seba, Impulsive fractional differential equations in Banach spaces, Electronic Journal of Qualitative Theory of Differential Equations Special Edition I. 8 (2009), 1-14. 1

[7] M. Benchohra, B. A. Slimani, Existence and Uniqueness of solutions to impulsive fractional differential equations, Electronic Journal of Differential Equations. 10 (2009), 1-11. 1 .

[8] B. Bonilla, M. Rivero, L. Rodriguez-Germa, JJ. Trujillo, Fractional differential equations as alternative models to nonlinear differential equations, Applied Mathematics and Computation. 187 (2007), 79-88. 1

[9] Y. K. Chang, Controllability of impulsive functional differential systems with infinite delay in Banach spaces, Chaos, Solitons and Fractals. 33 (2007), 1601-1609. 2.6

[10] Gisele M Mophou, Existence and uniqueness of solutions to impulsive fractional differential equations, Nonlinear Analysis. 72 (2010), 1604-1615. 1

[11] JH. He, Approximate analytical solution for seepage flow with fractional derivatives in porous media, Computer Methods in Applied Mechanics and Engineering. 167 (1998), 57-68. 1

[12] E. Hernandez, D. O'Regan, K. Balachandran, On recent developments in the theory of abstract differential equations with fractional derivatives, Nonlinear Analysis: Theory, Methods and Applications. 73 (10) (2010), 34623471. 1

[13] R. Hilfer, Applications of Fractional Calculus in physics, Singapore: World Scientific; 2000. 1

[14] V. Lakshmikantham, Theory of fractional differential equations, Nonlinear Analysis, Theory methods and Applications. 60 (10) (2008), 3337-3343. 1

[15] G. M. Mophou, G. M. N'Guéréata, Existence of mild solution for some fractional differential equations with nonlocal conditions, Semigroup Forum. 79 (2) (2009), 322-335. 1

[16] Mouffak Benchohra, Samira Hamani, The method of upper and lower solutions and impulsive fractional differential inclusions, Nonlinear Analysis:Hybrid Systems. 3 (2009), 433-440. 1

[17] B. N. Sadovskii, On a fixed point principle, Functional Analysis and its Applications. 1 (2)(1967), 74-76. 2.5

[18] Xianmin Zhang, Xiyue Huang, Zuohua Liu, The existence and uniqueness of mild solutions for impulsive fractional equations with nonlocal conditions and infinite delay, Nonlinear Analysis:Hybrid Systems. 4 (2010), 775-781. 2 